\title{
Spin-up problems of stratified rotating flows inside containers
}

\author{
Peter W. Duck $\dagger$
}

School of Mathematics, University of Manchester, Manchester M13 9PL, UK

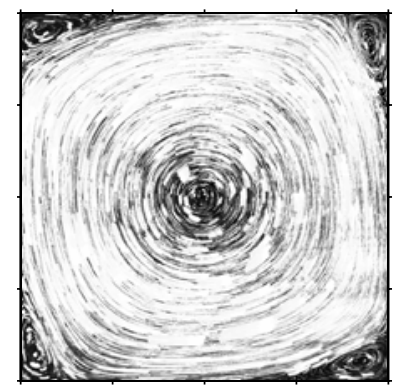

Rotating, stratified flows are important in a wide variety of both geophysical and engineering applications. Whilst 'steady state' flows of this type are generally very simple (in effect, rigid body rotation), the effect of abruptly altering (even a little) the rotation rate can induce significant temporal flow disruptions, made all the more complicated when the fluid is bounded inside a closed finite container, a problem studied both experimentally and theoretically by Foster \& Munro (J. Fluid Mech., this issue, vol. 712, 2012, pp. 7-40).

Key words: boundary layer structure, rotating flows, stratified flows

\section{Introduction}

Rotating flows subject to an abrupt change in rotation rate have been the subject of much research activity, both of an experimental and theoretical/computational nature, over many decades. There are many physical and engineering applications for such flows, including turbomachinery, centrifuges, the dynamics of components of spacecraft and in astrophysics (a detailed discussion of the dynamics within the Sun's core can be found in Benton \& Clark 1974). One of the earliest theoretical studies of this type, for homogeneous (i.e. non-stratified) and bounded flows, was that of Greenspan \& Howard (1963), work that has formed the basis for numerous later theoretical studies. In essence, after a (small) impulsive change to the rotation rate of a closed container containing a viscous fluid, thin so-called 'Ekman layers' form on the endwalls of the container, after just a few rotations of the container. These are thin layers, predominantly steady in nature, involving a balance between the pressure gradient, Coriolis force and the viscous drag. With time, in the axisymmetric case, fluid in the upper (lower) Ekman layer moves towards the vertical sidewall, and then is transported down (up) the sidewall in the ' $1 / 4$ ' and ' $1 / 3$ ' Stewartson layers (Stewartson 1957). The upper and lower Stewartson layers then collide to form a shear layer, directed towards the container centreline, leading to two meridional-plane circulation regions. An early review of work in this area is given in Benton \& Clark (1974), whilst a more recent review is that of Duck \& Foster (2001).

The interaction between stratification and rotation leads to an even more complex, rich and interesting assortment of physical phenomena. The earliest (experimental) work on the stratified spin-up of fluids in containers was that of Ekman (1906). Stratification has the effect of inhibiting the transport of vorticity contained in wall

$\dagger$ Email addresses for correspondence: duck@ma.man.ac.uk 


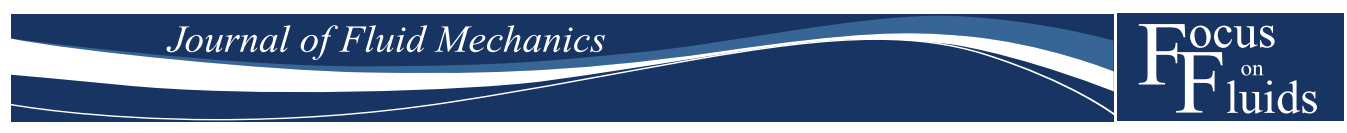

(a)

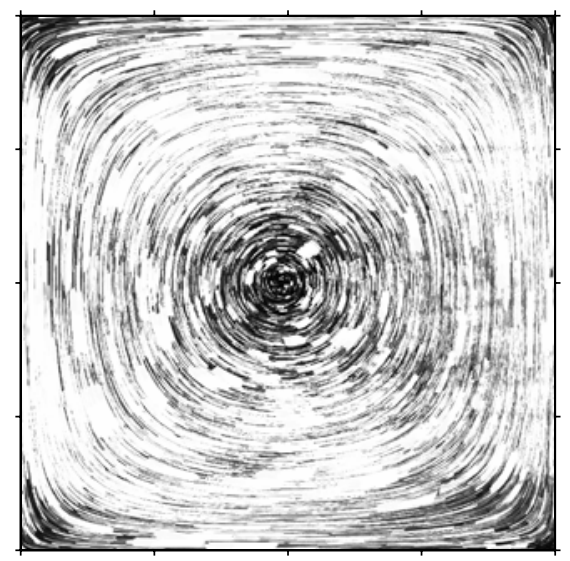

(b)

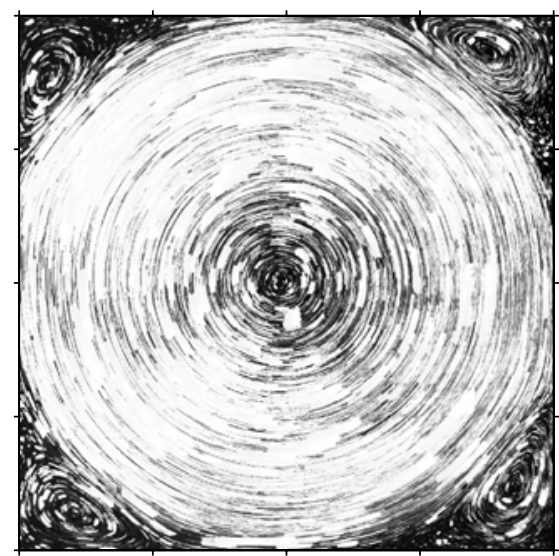

FIGURE 1. Streakline images from experiment C of Foster \& Munro (2012): (a) $\Omega t^{*}=8.6$; (b) $\Omega t^{*}=120$.

layers into the main body of the flow, and a number of theoretical and experimental papers have been published, primarily dealing with flows inside cylindrical containers, where axisymmetry is clearly a simplifying factor. A detailed history and fuller description of this class of flow can be found in Duck \& Foster (2001). The corresponding problem of spin-up of stratified flows inside rectangular containers was investigated by van Heijst, Davies \& Davis (1990) (leading on from the homogeneous case, studied by van Heijst 1989). The paper by Foster \& Munro (2012, this issue) leads on from this, employing a combined experimental and theoretical approach.

\section{Overview}

The loss of symmetry that must occur with square containers inevitably leads to much more complex spin-up flow structures, not least exacerbated by the nature of the flow in the vicinity of the corner regions. The earlier work of van Heijst et al. (1990) (which was also a combined experimental and theoretical study) used a flow visualization procedure involving small $(\sim 0.5 \mathrm{~mm})$ neutrally buoyant polystyrene particles suspended in the fluid, in which the motions of the particles were captured by cameras moving in the rotating frame, with the container. The experimental work of Foster \& Munro (2012) uses the more modern particle imaging velocimetry (PIV) technique, where tiny tracer particles are suspended within the fluid, which are assumed to follow (but not affect) the local fluid flow. The motion of the seeding particles is captured by a digital camera, and the resulting images analysed on a computer (using signal-processing and correlation techniques) to calculate displacement vectors and hence the velocity field of the fluid. Time-exposures of the captured images can also be used to generate 'streaklines' showing the particle trajectories. Two streakline images taken from Foster \& Munro (2012) are shown in figure 1, where the image at the later time shows clear signs of the corner eddy cells.

The initial-time regime in the bulk of the flow is two-dimensional, provided that the stratification is sufficiently strong, as was established by van Heijst et al. (1990) and is the starting point for the theoretical aspect of the investigation of Foster \& Munro 
(2012). The associated streamline pattern is of a single cell spanning the entire domain, and as a consequence is a region of uniform vorticity (Batchelor 1958) and so the interior of the flow is governed by Poisson's equation. One of the major contributions of the work of Foster \& Munro (2012) is putting theory on to a much more rigorous footing than previous work. This initial regime is shown to be valid for (dimensional) time $t^{*}=O\left(\Omega^{-1}\right)$, where $\Omega$ is the (initial) rotation rate of the container, and the Ekman number $E=v / \Omega L^{2}$ (which, following much work in this area is assumed small - this is an important flow regime, due to the general smallness of $v$, the kinematic viscosity of the fluid); here $L$ is a characteristic length scale of the container.

Following on, Foster \& Munro (2012) then consider a somewhat longer time scale, namely $t^{*}=O\left(E^{-1 / 2} \Omega^{-1}\right)$, i.e. the flow regime after that considered by van Heijst et al. (1990). In this case, the interior of the flow not only becomes dependent on the vertical coordinate, but is also 'fed' by so-called Ekman suction - effectively transpiration (suction) of fluid from the interior flow into the Ekman layers lying on the upper and lower container surfaces. The solution to this problem is complicated, but Foster \& Munro (2012) are nonetheless able to construct analytic solutions.

The next important contribution of Foster \& Munro (2012) is their analysis of the sidewall layers. This highlights a significant difference with the axisymmetric cases, namely that unlike the latter, the tangential and normal velocity components on the sidewalls in the regime $t^{*}=O\left(E^{-1 / 2} \Omega^{-1}\right)$ are no longer zero, and hence wall layers are necessary in order to satisfy the usual wall boundary conditions (no slip, impermeability). It is here that the smallness of the Rossby number $\epsilon$ (the ratio of the change in rotation rate to the initial rotation rate) is invoked in order to maintain the linearity of the flow structure within these layers, which then take the form of 'Rayleigh' layers (involving a balance between acceleration and viscous terms, and a pressure gradient - the absence of inertia terms is due to the assumed linearity).

It is at this point that Foster \& Munro (2012) make their most important contribution/observation: a comparison between their experimental measurements (made with PIV) and theory, specifically in the aforementioned later $t^{*}=O\left(E^{-1 / 2} \Omega^{-1}\right)$ regime, by constructing a composite solution from the asymptotic results (i.e. a combination of the interior solution with the Rayleigh layer solution, along the lines described by van Dyke 1964). Detailed comparisons are made with experimental observations along the symmetry axes, specifically for the horizontal velocity component; the correlation between experiment and theory is remarkable. This is all the more impressive because, as noted in the paper, strictly for the asymptotic theory to be truly applicable, it is necessary that $1 \ll E^{1 / 2} / \epsilon \ll 1 / \epsilon$, whilst table 1 in the paper indicates that $0.06 \lesssim E^{1 / 2} / \epsilon \lesssim 0.31$ in the experimental setups. Indeed, a good asymptotic theory is one that works when it should not!

Returning to consider further theoretical aspects of the paper, the authors then go on to consider the nature of the sidewall layer at (still) later times, specifically when $t^{*}=O(1 /(\Omega \epsilon))$. The key observation from this is that even though the Rossby number has been assumed small, ultimately, within this later time scale, inertia effects must play a leading role in the flow physics, and hence nonlinearity is important. The result is that the flow close to the sidewalls is governed by the full unsteady Prandtl-type boundary-layer equations. Although these equations (which are nonlinear, involving a balance between inertia, acceleration and viscous terms, together with the tangential pressure gradient, as calculated from the interior solution) are not tackled in the paper, it is very clear that eddies must form in the corner regions of the flow (indeed, corner eddy cells were clearly observed in the experiments, see figure $1 b$ ). This is inevitable, given that the pressure gradient along the sidewall boundary layers is adverse over half 
of the section of each wall. The implication of this is that as well as reversed flow occurring, a finite-time singularity, at some finite wall location must also occur, and this is likely to be of the van Dommelen \& Shen (1980) variety, with a singularity involving the displacement thickness of the boundary layer. Indeed, such breakdowns are quite common, although the analytic description of flow regimes beyond such breakdowns remains a fundamental but challenging problem for theoreticians.

\section{Future}

It is clear that the next phase in considering this class of problem is to fully incorporate nonlinear effects. The Prandtl-boundary-layer problem, posed by Foster \& Munro (2012) is certainly worthy of attention. The key result from this would be the time and location of the 'eruption' of the boundary layer, and this would give two very tangible values to compare with experiment. As noted above, the flow regime(s) following these types of breakdown remain a major difficulty, but a rigorous asymptotic description is still lacking, and would have application far beyond problems of this type (in fact to boundary-layer/high-Reynolds-number theory, in general).

A further line of study, which is perfectly feasible these days, would be a direct numerical simulation (DNS) of the full governing equations (specifically equations $(3.5 a-c)$ in Foster \& Munro 2012). These should be three-dimensional in nature, but even the linearized (based on small Rossby number) results would be of interest. Other flow geometries and configurations should also prove to be interesting directions of investigation. To conclude, the work of Foster \& Munro (2012) gives us a much clearer picture of the flow inside non-axisymmetric, rotating containers, when the rotation rate is abruptly altered, both from the experimental and the theoretical perspective.

\section{References}

BAtCheloR, G. K. 1958 On steady laminar flow with closed streamlines at large Reynolds number. J. Fluid Mech. 1, 177-190.

Benton, E. R. \& Clark, A. 1974 Spin-up. Annu. Rev. Fluid Mech. 6, 257-280.

VAN Dommelen, L. L. \& SHEN, S. F. 1980 The spontaneous generation of the singularity in a separating laminar boundary layer. J. Comput. Phys. 38, 125-140.

Duck, P. W. \& Foster, M. R. 2001 Spin-up of homogeneous and stratified flows. Annu. Rev. Fluid Mech. 33, 231-263.

VAN DyKe, M. D. 1964 Perturbation Methods in Fluid Mechanics. Academic.

Ekman, V. W. 1906 Beitäge zur theorie der Meeresströmungen. Ann. Hydrograph Mar. Met. 2, $1-50$.

Foster, M. R. \& Munro, R. J. 2012 The linear spin-up of a stratified, rotating fluid in a square cylinder. J. Fluid Mech. 712, 7-40.

Greenspan, H. P. \& HowARD, L. N. 1963 On the time dependent motion of a rotating flow. J. Fluid Mech. 17, 385-404.

VAN HEIJST, G. J. F. 1989 Spin-up phenomena in non-axisymmetric containers. J. Fluid Mech. 206, 171-191.

VAn Heijst, G. J. F., Davies, P. A. \& Davis, R. G. 1990 Spin-up in a rectangular container. Phys. Fluids A 2, 150-159.

Stewartson, K. 1957 On almost rigid rotation. J. Fluid Mech. 3, 17-26. 